\title{
The Value of a General Practitioner Completed Pre-employment Medical Questionnaire in Recruit Selection
}

\author{
Maj J P Owen \\ $M B, C h B, A F O M, R A M C$
}

Army Personnel Research Establishment, Ministry of Defence, Farnborough, Hants GU14 6TD

SUMMARY: A General Practitioner (GP) completed, fee earning, medical questionnaire was obtained for 444 potential Army recruits and compared with the examination findings of Army Careers Information Office Medical Officers (ACIO MOs), recorded on the FMed 1. It was found that $34(8 \%)$ of the GPs reported a condition that' precluded military service. Fourteen of those candidates denied the medical problem on their FMed 1 and remained undetected. Twenty candidates declared their condition but only 6 were rejected, 6 were referred for a specialist opinion and 8 were passed fit. This represents a $60 \%$ detection rate among declared problems and nil among concealed ones.

If a GP questionnaire were available for all candidates and the same detection rate achieved, there would be a $1.8 \%$ increase in rejections at the pre-employment medical selection. The GP questionnaires would cost approximately $\mathbf{f 4 0 0 , 0 0 0}$ each year. There should however be a compensating reduction in individual requests for GP reports by the ACIO MOs and inappropriate specialist referrals. A drop of $1.8 \%$ in recruit medical discharges would save $\$ 5,700,000$ annually. This study recommends obtaining a GP medical questionnaire for all candidates applying to join the Army.

\section{Introduction}

When potential recruits apply to join the Armed Forces they undergo a medical examination as part of the overall selection process. This is to ensure the applicants are medically fit for service and that service will not be detrimental to their health.

Under Section 2 of the Health and Safety at Work etc Act 1974 (1), employers have a duty to ensure, as far as is reasonably practicable, the health, safety and welfare of all their employees, one of the areas of application being the health screening of workers prior to employment. Screening fulfils a number of functions, primarily that of trying to ensure that the employees are fit for their jobs and that their health will not suffer as a result of taking the job. The Health and Safety Executive (HSE) offers further guidance on pre-employment health screening (2) and several papers have been published on the subject (3, $4,5)$

The reasons for pre-employment screening can be summarised as:

a) To ascertain an individual's fitness to perform a job.

b) To ensure that the individual's health will not suffer from the job or be a risk to other employees.

c) To protect the employer where long-standing disability or ill-health may lead to repeated loss of time from work.

d) To ensure that others are not placed at risk by an employee's ill-health. This may be a legal requirement e.g. HGV drivers (6), pilots (7), divers and compressed air workers (8).

e) Secondary benefits include occasional discovery of previously undetected illness and introducing employees to the medical service provided by the employer.
In the case of the Army the employees are soldiers and $7-$ have to be able to work under arduous conditions. Under 0 T field conditions and in combat, the health and safety of the $\frac{\mathbb{Q}}{\square} \frac{\mathrm{D}}{\mathrm{C}}$ soldiers is very much dependant on mutual support, ill- $\mathbb{\mathbb { Q }}$ ness or debility in individuals may cause a real risk to themselves and their colleagues.

Military tasks may also be governed by the civilianlegal requirements already mentioned, e.g. pilots (7), HGV drivers (6), radiation workers (9), cooks and food handlers (10).

The nature of a soldier's job means that he or she will be in contact with potentially lethal weapons. There is therefore a responsibility on the employer to ensure that the soldier will not be a risk to others (or himself), which raises the need for mental as well as physical fitness for the job.

To achieve this aim in the Army candidates undergo a medical selection process which starts at the Army Careers Information Office (ACIO), where applicants complete a medical questionnaire (FMed 1). They then undergo a medical examination from the ACIO Medical Officer (ACIO MO) which is recorded on the same form. Guidance on the criteria for this examination is given in the Joint Service Publication 346 (JSP 346) (11).

The ACIO MO carrying out pre-employment examinations has not previously seen the applicants and does not have routine access to any previous hospital or GP's medical notes. This information can only be obtained with the prior, informed and freely given consent of the applicants.

When completing the FMed1 applicants declare their truthful and full completion of the questionnaire and assent to the Service Medical Authorities having access to any previous medical records. However there are a number of reasons why this is not always the case: 
a) Applicants genuinely do not know that they have or have had a particular condition or medical problem. It may have occurred in early childhood or previous medical attendants have not disclosed the full diagnosis.

b) Deliberately withholding information as they may be worried or know that it would prejudice their selection.

c) Not understanding the questionnaire properly.

d) Genuinely forgetting some aspects of their medical history.

If the ACIO MO suspects a problem from the questionnaire or examination he may obtain extra information from the applicant's past medical attendants. A specialist opinion may also be sought in some cases. This takes time and often involves several trips to the ACIO for the applicant, as well as the expense of specialist reviews. There is also evidence that the specialist reviews are often not appropriate as the problem presented precludes military service, according to JSP 346 (12). One factor compounding this may well be the lack of past medical information available to the ACIO MO.

There are several conditions which preclude military service which only occur intermittently, with apparent normal health in between. e.g. epilepsy, asthma, emotional instability. In these cases, if the applicants conceal their history, the examination will usually be normal. Therefore the ACIO MO will have no reason to suspect any precluding conditions and the applicant will be declared fit to commence training.

The nature of these conditions is that they recur and seriously interfere with the soldiers' ability to carry out their duties. Anyone who manages to evade detection at selection then becomes a liability to themselves and their colleagues. They also cause a financial and training burden in manpower wastage due to medical discharges.

Jefferson (13) reported that recruit medical wastage in 1988 accounted for $16.7 \%$ (897 cases) of all causes of discharge from the Army, at an estimated cost of $£ 14,318,000$. The average duration of service was 148 days. Over half of the recruits were discharged under the authority of Queen's Regulations 9.381 (14) which deals with defects of enlistment procedure. It states that a soldier may be discharged if it is found after enlistment that he or she is medically unfit on the basis of a condition that pre-existed at his or her enlistment.

The earliest detection of unsuitable applicants ensures that wastage is kept to a minimum. One way of increasing the effectiveness of the medical selection is to have more information on the applicants at the time of the selection medical examination.

\footnotetext{
Aim

The aim of this study was to investigate the use of a General Practitioner (GP) completed pre-employment screening questionnaire as a means of predicting potential medical discharges.
}

\section{Method}

The Director of Army Recruiting identified 4 ACI $\stackrel{\text { S }}{\text { S }}$ with a high throughput of potential recruits and the staffat these centres were briefed on the purpose of the study with 250 consent forms being distributed to each office.

Once all documentation of the potential recruit ws completed the ACIO arranged for an entrance medicgl examination to be carried out. At this time the candidat were asked for their consent for the Senior Medical Of cer at the Army Occupational Health Research Unft (AOHRU) to approach their GP for information regardifpg any previous medical history. All potential recruits w informed that participation in the study was voluntary. They were also told that the study was independent their enlistment and could not affect it. The consent forms were then taken away for signature by the potent recruits and returned to AOHRU in a postage paid enve lope.

A pre-employment screening questionnaire was designed at AOHRU, based on a typical life insurance medical questionnaire. On receipt of the signed consent form, the screening questionnaire was sent to the GP cotsplete with a reply paid envelope. Once the questionmaire was returned to AOHRU, the normal payment for is ance medical questionnaires ( $£ 20$ per completed for was sent to the GP.

The ACIO MO completed the examination of the recruit and having completed an FMed1, copied it $\overrightarrow{00}$ AOHRU. The ACIO MO did not know whether or ne the recruit had opted to take part in the study and did nofsee the completed GP questionnaire.

The GP questionnaires were examined by an Array Occupational Health Physician and classified according कo the criteria given in JSP 346 . Only those cases wepe rejected where the information given unequivocally fend outside the requirements of JSP 346. All the cases whete the GP expressed some doubt over a diagnosis or severity of a problem were accepted and noted for probable se cialist referral. The GP questionnaires were analysed agid loaded onto a computer database before the FMed1s avoid a possible introduction of bias. The data from the two sources was then compared.

The headings under which the questionnaires we compared were taken from those listed in Section 2 of FMed 1, where the details of the past medical history a recorded in a standard format, the information given ofn the GP questionnaires being allocated to the apprôpriate section, which enabled a direct comparisan between the information given from the two different sources.

Those GP questionnaires which indicated a problem falling outside the JSP 346 requirements were noted afol compared with the PULHHEEMS grade given by the ACIO MO. The error rate in both the completed \& questionnaires and FMed1s was also recorded and coffipared. 


\section{Results}

A total of 1000 consent forms were distributed to the four ACIOs, with 250 forms being given to each office. Only 672 were distributed to applicants in the time available to the study.

Five hundred and fifty nine forms were returned to APRE, giving a response rate of $83 \%$ for completed consent forms. All of these were then sent to the candidates' GPs and 504 completed questionnaires were returned to APRE, a response rate of $90 \%$. There were 70 questionnaires returned which could not be matched to a FMed 1 , for the reasons shown below:

a) 40 potential recruits did not return for an ACIO Medical Examination.

b) 13 recruits were enlisted but the FMed1s were not copied to AOHRU.

c) 1 potential recruit moved ACIO during selection.

d) No record could be located at the ACIOs for 16 potential recruits.

There were problems with the quality of 73 of the returned GP questionnaires and 120 of the FMed1s, as shown in Table 1 . This resulted in 444 records held with matching FMed 1 and GP questionnaires. Three hundred and twenty three $(73 \%)$ candidates were declared fit by the ACIO MOs, with $85(19 \%)$ specialist referrals or requests for extra GP information being made and 34

Table 1

Quality Problems on GP Questionnaires and FMed1s

\begin{tabular}{lll}
\hline & GP questionnaires & FMed1s \\
\hline a) Illegible sections & $25(6 \%)$ & $8(2 \%)$ \\
b) Blank sections & $34(7 \%)$ & $93(21 \%)$ \\
c) Wrong patient & $14(3 \%)$ & - \\
d) Wrong grade given & - & $19(4 \%)$ \\
\hline
\end{tabular}

$(8 \%)$ of the candidates being rejected as unfit for service. Some of these had also been reviewed by a military medical specialist or had reports requested from their GP.

Examination of the GP questionnaires revealed 251 $(56 \%)$ candidates with no relevant problems declared. One hundred and fifty nine, $(36 \%)$ of the candidates warranted referrals and $34(8 \%)$ should have been automatically rejected. Details of the causes for rejection are given in Table 2. Further examination of these candidates revealed that $20(59 \%)$ declared the problem on the FMed 1. Of these 8 were passed as fit, 6 were referred and 6 were rejected. The remaining $14(41 \%)$ candidates denied any problems on the FMed 1, with 10 of them being passed as fit and 4 being referred for unrelated problems, while none were rejected. The overall outcome was 18 $(53 \%)$ declared fit, $9(26 \%)$ referred and $7(21 \%)$ rejected.

The prevalent problem declared by the GPs was asthma. There were 42 cases of which 12 (29\%) should
Table 2

\begin{tabular}{lll}
\multicolumn{3}{c}{ Number of Rejections by Cause } \\
\hline Condition & JSP 346 Reference & Number \\
\hline a) Asthma & Para. 0418 & 12 \\
b) 'S' (Stability) & Para. 0489 & 9 \\
c) Nerv. Sys. Migraine & Para. 0438 & 5 \\
", Seizure & Para. 0437 & 2 \\
d) Knee Pain & Para. 0481 & 2 \\
e) Hearing/TM probs. & Para. 0406 & 2 \\
f) Back Pain & Para. 0456 & 2 \\
g) Renal Prob & Para. 0421 & 1 \\
h) Splenectomy & Para. 0458 & 1 \\
i) Enuresis & Para. 0424 & 1 \\
j) Peptic ulcer & Para. 0432 & 1 \\
Four candidates had two reasons for automatic rejection. \\
\hline
\end{tabular}

have been rejected according to JSP 346. Analysis of $\overrightarrow{\vec{P}}$ those warranting rejection revealed that 9 candidates did $ㅇ$ declare asthma or wheeze on the FMed1. Of these 1 was $\omega$ declared unfit and 8 were referred by the ACIO MO. However 3 candidates denied asthma on the FMed 1 and were passed fit by the ACIO MO.

It was noted that there were 7 candidates who declare asthma or wheeze on the FMed1 which was not matcheg by the GP questionnaires. Of these candidates, 3 deni ever having seen their GPs for their wheeze, 1 GP queş $\overrightarrow{0}$ tionnaire was incorrectly completed and invalid, 1 क्ष reported Perennial Rhinitis and Allergy testing. The remaining 2 GPs had known their patients for sever years but did not make particular comment. The outcomie of the ACIO MO examination for these candidates were 1 declared fit and 6 referred.

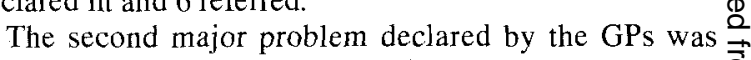
emotional stability, classified under the ' $S$ ' factor in the PULHHEEMS grading(15). Twenty one cases were declared of which $9(43 \%)$ should have been automatically rejected according to JSP 346. Only 3 of those candidates declared their problem on the FMed1; 2 were passed as fit (1 having taken an overdose, 1 requiring in patient treatment for behaviour problems) and 1 was rejected (being anorexic and using illicit drugs). There $\stackrel{\mathbb{D}}{\stackrel{D}{D}}$ were 6 candidates who denied any mental instability on the FMed 1 and all of them were passed as being fit. The concealed problems were; depression still under drug therapy in 2 cases; depression still under psychotherapy in 0 2 cases; illicit drug and solvent abuse in 2 cases; behaviour problems requiring in-patient treatment in 1 case.

It was noted that there were 8 candidates who declared $\frac{D}{0}$ an emotional stability problem on the FMed 1 but were not commented on in the GP questionnaires. Five of these $N$ were declared fit by the ACIO MO, 2 were referred and $1 \mathrm{~N}$ was not given a ' $\mathrm{S}$ ' grade but was rejected by the ACIO N MO for backache problems. 


\section{Discussion}

The study was well accepted by potential recruits at the ACIOs with over $80 \%$ of the consent forms being returned to APRE for passing on to the GPs. The response from the GPs was also fairly good at $90 \%$, though it should be borne in mind that $10 \%$ did not respond despite being offered a standard payment for the completed report. No second calls were made in this study to try and investigate this group of GPs.

Even though the ACIOs were chosen for their high throughput of applicants, only two of them managed to distribute all of their allocated consent forms before the study was closed. Therefore, once GP non-compliance had also been accounted for, the anticipated $n$ of 1000 was reduced to 444 .

Whilst the ACIO MOs did not see the completed GP questionnaires and did not know which applicants had responded, they did know that their ACIO was involved in the study. This may have caused a change of practice, though this seems unlikely as the rejection rate of $8 \%$ from the FMed1s in this study matches that reported by Dignan (16) in 1992.

The prevalent causes for rejection from the GP questionnaires were asthma (12 cases), emotional instability ( 9 cases) and nervous system problems of migraine or seizures ( 7 cases). This is different from Dignan's report where orthopaedic problems accounted for $34.5 \%$ of the rejections (16). However the criteria for exclusion in JSP 346 are far more definite for respiratory problems (JSP 346, paras. 0416-0420) than orthopaedic ones (JSP 346, paras. 0452, 0453, 0455-0457, 0468-0482) and therefore easier to apply to a questionnaire where the candidate cannot be examined. In the case of asthma JSP 346 para. 0418 is clear that new entrants 'with current asthma or a history of wheezy bouts requiring treatment during the last four years are graded P8'. The orthopaedic problems, in general, depend on the degree of mobility and function retained and require an assessment by a MO familiar with the military needs.

The problem of candidates who conceal their past history to the ACIO MO is shown in that 3 of the candidates with asthma or wheeze who warranted automatic rejection from the GP questionnaire did not declare any problem on the FMed 1 and had a normal examination, being passed fit. In the case of mental instability a similar picture is shown, with 6 candidates being passed fit by the ACIO MO, having concealed a past history of mental instability which would have resulted in automatic rejection. In both of these categories there were a few candidates ( 7 asthma or wheeze and 8 mental instability) who declared a problem on the FMed1 which was not corroborated by the GP. The problems tended to be relatively minor, with 3 of those declaring wheeze stating that they had never seen their GP with the problem, whilst the mental instability problems were recorded as 'Wary of Heights', 'One week of depression five years ago' and 'Early school behaviour problems'. None of those candidates who declared a prob- lem to the ACIO MO but without GP corroboration faile the examination.

It is of note that out of 34 of those candidates who ha $\$$ problems that precluded military service as declared bo the GP, 18 (53\%) were passed as being fit by the ACI MO on the information that was available. Moreover though 20 candidates did declare their problem to the ACIO MO, 8 were still passed as fit (though one of theso was later rejected for an unrelated problem), 6 wer흥 referred for further examination and 6 were rejected Effectively only $60 \%$ of those candidates with problem precluding service which were declared to the ACIO MQ were detected as such.

If the GP reports on all candidates had been availabl $\vec{D}$ to the ACIO MOs another 14 cases for rejection would have been seen. Assuming a $60 \%$ detection rate and resu㕄 tant action, another 8 cases would be rejected. This repre sents a $1.8 \%$ increase in detection rate at the pre-employ? ment medical examination.

Jefferson (13) reported that approximately $20,00 \mathrm{~A}$ applicants were sent to training establishments each yeap A $1.8 \%$ improvement in the selection translates to as annual increase in detection of 360 medically unfit candfi dates each year. Using the GP questionnaires with a associated $£ 20$ fee would result in an approximate cos?tof $£ 400,000$. However this should be compared to the a品星 age cost of $£ 15,900$ for each recruit medically discharge after joining the Army (13). Avoiding 360 medical \&is charges would prevent the wastage of $£ 5.7$ million. ए

There were some problems with the quality of $\$ h{ }^{\circ}$ reports, both from the GPs and the ACIO MOs. With hhe GPs, legibility and missing out sections of the report the most common faults, possibly due to the freestyle Eys out of the questionnaire. It was evident, on comparing the GP reports with the details on the FMed1s, that a number of the reports had been completed for the wrong patien This was shown by the wrong date of birth being giver records held before the candidates birth and operations accidents inappropriate to the candidate's age. Alse despite being paid $£ 20$ for each completed form, $10 \%$ 迎 the GPs did not respond to the request for a repors Though it was not done in this study, these problem could be resolved with a second call to the GPs to clarif and recover their statements.

\section{Conclusions}

The use of a GP questionnaire is effective in improvin the detection rate of medically unsuitable cases and should be used in addition to the ACIO pre-service mes ical examination for all potential recruits .

There will be a cost to be met from getting the informa tion from the GPs. However this should be partly cont pensated for by the reduction in number of requests for GP reports from the ACIO MOs during the selectio process. At the time of this study the cost of a GP repont requested by the ACIO MO was $£ 16.40$ for a limiten report from the notes and $£ 26.00$ for a full report ang 
examination (10). There is also the potential for saving by preventing inappropriate referrals to Hospital Specialists, when the GP has documented the degree of an existing illness such as asthma.

The major benefit in terms of reducing recruit wastage should be seen in a reduction in the number of recruits who evade detection at the initial medical examination only to be medically discharged at a later date.

\section{Acknowledgements}

The assistance of the 4 ACIOs and their MOs who agreed to take part in this study is gratefully acknowledged. At the AOHRU the help given by Cpl Campbell, RAMC is acknowledged in tracing FMed1s and collecting the reports from the GPs.

\section{REFERENCES}

1. Health and Safety at Work etc Act 1974. HMSO, London 1974.

2. Health and Safety Executive. Guidance Note MS20 (May 1982), Pre-employment Health Screening. HMSO, London 1982.

3. DUNCAN K P. Examining Examinations: Pre-employment Medical Examinations. J Soc Occup Med 1989; 39: $152-153$.

4. Kelman G R. The Pre-employment Medical Examination. Lancet 1985; ii: 1231-1233.

5. FLOYD M, EsPIR M L E. Assessment of Medical Fitness for Employment: The Case for a Code of Practice. Lancet 1986; ii: 207-209.
6. Raffle P A B. Medical Aspects of Fitness to Drive: a Guide for Medical Practitioners. 4th ed. London. Medical Commission on Accident Prevention, 1985.

7. International Civil Aviation Organisation. Annex 1 to the Treaty on International Civil Aviation. 7th ed. Montreal: International Civil Aviation Organisation, 1982.

8. Diving Operations at Work Regulations 1981 (SI 1981 no 399). HMSO, London.

9. Ionising Radiation Regulations 1985. HMSO, London.

10. The Food Hygiene (General) Regulations 1970 (SI 1970 no 1172). HMSO, London.

11. Joint Service Publication 346 Pulhheems. A Joint Service System of Classification. Ministry of Defence. 1992.

12. Johnston J H. Potential Recruits in medical Outpatients - An Audit. J R Army Med Corps 1990; 136: 102-104.

13. JEFFERSON $T \mathrm{O}$. An Investigation into Regular Recruit Wastage from the British Army, 1988. JR Army Med Corps 1990; 136: 138-145.

14. The Queen's Regulations For The Army 1975. HMSO, London 1975.

15. Pulhihems Administration Pamphlet 1987. Army Code No. 13371.

16. Dignan A P. A Decade of Experience of Examining Candidates for Entry to the Army. J R Army Medo Corps 1992; 138: 19-22.

17. Civil Management Specialist Branch (2C1A), Bath. Letter, Reference D/CM(S) 2/36/5/3, dtd 27 Apr 92. 